\title{
Comparison of patient outcomes in academic medical centers with and without value analysis programs
}

\section{Adrienne S Murray \\ Michael Griswold \\ Imran Sunesara \\ Ed Smith}

University of Mississippi Health Care, University of Mississippi Medical Center, Jackson, MS, USA
Correspondence: Adrienne S Murray Supply Chain Management, University of Mississippi HealthCare, 2500 North State St, Room C782, Jackson, MS 39216, USA

$\mathrm{Tel}+\mathrm{I} 6018155436$

$\mathrm{Fax}+\mathrm{I} 6018153758$

Email asmurray@umc.edu
This article was published in the following Dove Press journal:

Journal of Healthcare Leadership

3I August 2012

Number of times this article has been viewed

Background: Value analysis is the science of balancing the mandate to deliver high-quality clinical outcomes with the necessity to drive down costs in order to thrive in the challenging economics of health care. This study compared average length of stay, direct cost, morbidity, and mortality across the cardiology, cardiovascular, neuroscience, and orthopedic service lines, in academic medical centers with and without value analysis programs (VAPs). The basic question was, "Do academic medical centers with VAPs have lower average length of stay, better morbidity and mortality rates, and lower overall supply costs?"

Methods and results: The clinical data base/resource manager (CDB/RM) of the University HealthSystem Consortium was utilized as secondary data for this study. Reports from the CDB/ RM were generated from 2006 to 2011. Continuous variable differences across VAP status were examined using Wilcoxon two-sample tests. Primary analyses used multilevel linear mixed model methods to estimate the effects of VAPs on primary outcomes (average length of stay, cost, morbidity, mortality). Association components of the linear mixed models incorporated random effects at the hospital level and robust, Huber-White, standard errors were calculated. There was no significant difference for average length of stay, direct cost, morbidity, and mortality between academic medical centers with and without VAPs. However, outcomes were not noted to be substantially worse.

Conclusion: Numerous case studies reveal that aggressively active VAPs do decrease hospital cost. Also, this study did not find a negative impact on patient care. Further studies are needed to explore the benefits of value analysis and its effect on patient outcomes.

Keywords: value analysis, average length of stay, morbidity, mortality, health care costs, supply chain

\section{Background}

In the US, value analysis began in 1947 at General Electric. The founding father was Lawrence D Miles. While at General Electric, Miles was responsible for identifying, negotiating, and acquiring key materials that were scarce. In many instances, he found the situation to be in dire straits and aimed at getting product functions met by alternative means. More often than not, Miles found a way to meet product functions via substitutes that provided equal or better performance at a lower cost. Mile's success at General Electric prompted private industry to adopt this concept. In 1954, the US Department of Defense Bureau of Ships (now the Navy Ships System Command) implemented a formal program and called it "value engineering". By 1961, value engineering had been established throughout the US Department of Defense. Its applications have been used in many industries, including the automobile, railways, metal, electrical 
equipment, and service sectors. It was not until the $1990 \mathrm{~s}$ that value analysis first appeared in health care. ${ }^{1}$

\section{Introduction}

According to Marmor et al, the health care system in the US faces many challenges, with cost and quality being key concerns. Health outcomes in the US are not exceptional when compared with other wealthy nations, yet the US spends more than any other country on medical care. ${ }^{2}$ Emanuel affirms that, compared with total US health care expenditure, there are little to no established resources or efforts to determine which technologies, products, or interventions are most effective. ${ }^{3}$ Americans spend more than \$2 trillion on health care annually, and over $\$ 100$ billion is spent on research and development as well as the regulatory approval of emerging technologies. However, annual spending on technology assessment falls shy of $\$ 1$ billion, representing $0.05 \%$ of total US health care spending, according to Ford. ${ }^{4}$ Value-based purchasing in health care reform is fast approaching, and health care in the US will transition from payment by volume to payment for performance. This change will significantly impact how health care providers in the US will be paid. The current reimbursement system in the US is a "fee for service" model, whereby health care providers are paid based on the quantity of care provided. However, value-based purchasing will focus on the quality of care provided, as well as patient satisfaction, rather than the quantity of care that is provided. Also, supply costs are on the rise, and quality of care and patient safety have emerged as chief concerns for reimbursement. Health care accounts for more than $17 \%$ of the gross domestic product in the US, with more than $\$ 19$ billion being spent on efforts to reduce medical errors. ${ }^{3}$

Singh and Schneller state that as hospital administrators look to reduce operating expenses, many are turning to supply chain. The supply chain environment, especially value analysis, is a capable field for process improvement, considering that the arena of value analysis has not been effectively explored regarding cost savings and its impact on patient outcomes. ${ }^{5}$ At many academic medical centers, value analysis has become a tactical cost reduction instrument for health care executives who utilize its principles. ${ }^{6}$ The general objective of this study was to compare patient outcomes, specifically average length of stay, direct cost, morbidity, and mortality, in academic medical centers with and without a value analysis programs (VAP). Value analysis is the balance between the business savings side of health care and patient outcomes. Value analysis is also the methodical review of the value of goods and services, whereas the goal of value analysis is to deliver high-quality care at the lowest total delivered cost. Murphree advises health care executives to dissect each service and product purchased to comprehend its intrinsic value while exploring for possible modifications and specifications that could decrease costs and improve quality care, as well as the institution's bottom line. ${ }^{6}$ Yokl recommends that health care executives focus on the purging of inefficiencies and waste in services, products, and technologies. ${ }^{7}$ The Healthcare Financial Management Association emphasizes that supply chain savings via value analysis can be reinvested into the facility for, eg, clinical programs and capital equipment. ${ }^{8}$ Unfortunately, guidelines/protocols for product suitability in health care have never been established pertaining to the application of product(s) in the practice setting. ${ }^{9}$ This is one of the many reasons VAPs are on the rise.

Kirschner asserts that a growing number of physicians agree that we desperately need a tool that brings value and cost-effectiveness together and has explored the availability and use of data that compare cost and clinical outcomes. ${ }^{10}$ However, it is the physician preference items that are the most costly to hospitals. It is estimated that supply costs represent nearly $31 \%$ of the total hospital cost per case. The most expensive items are the ones for which physicians have a penchant. These items represent $61 \%$ of the total supply expenditure. Often a gap exists between the preference of the physician and the goals of the hospital for cost containment. ${ }^{9}$ According to Coye and Kell, physician preference governs the better part of new technology purchases, particularly by the physicians who are accountable for admissions of copious proportions. According to the National Coalition for Quality Assessment, long-term savings can be generated by small gains in quality, and health care quality is not equal. ${ }^{11}$ According to Thorpe, economists hypothesize that there are two basic approaches to slow the rising costs of health care. The first is to reduce spending on high-cost medical technologies and supplies that produce no additional benefits, and the second is to reduce spending on high-cost care that generates some health benefits but at a higher price. ${ }^{12}$ The ultimate standard for advocating the implementation of technology is based on the following scientific considerations. Is the new technology cost-effective when weighed against existing technologies? Does it enhance existing technologies? Do the health benefits of the new technologies outweigh their potential harm? ${ }^{13}$

\section{Value analysis programs: an example}

The structure of VAPs vary among hospitals, but the fundamentals of value analysis are essentially the same across 
academic medical centers. Most academic medical centers have product standardization committees and/or surgical services committees in which the value analysis facilitator aids. An example of a value analysis initiative is examining oral care kits in order to prevent ventilator-associated pneumonia. Ventilator-associated pneumonia increases morbidity, mortality, and average length of stay, and Centers for Medicare and Medicaid do not reimburse hospitals for treatment of this hospital-acquired infection. Even though this initiative could increase supply costs by an additional $\$ 100,000$ per year, the average cost of treating a patient who develops ventilatorassociated pneumonia is roughly an additional $\$ 40,000$. If two or three patients acquire ventilator-associated pneumonia, then the additional costs rapidly exceed the added costs for new oral care kits. The value analysis facilitator takes into consideration that the traditional kits do not provide visual cues that oral care is performed every 4 hours, and that the new kits are partitioned into segments which do allow for visual cues that oral hygiene is performed every 4 hours. The new kits also contain improved products. The value analysis facilitator collects information on best practices for ventilator-associated pneumonia, evidence-based research that reveals the effectiveness of oral care kits in reducing the risk of ventilator-associated pneumonia, and studies from nursing and respiratory journals which arm committee members with the information necessary to make an informed decision. In order to measure the results, ventilator-associated pneumonia rates would be tracked. If 16 ventilator-associated pneumonia cases are eliminated as a result of implementing the new oral care kits, this initiative would result in a cost avoidance of more than $\$ 587,000$, and would also reduce average length of stay, morbidity, and mortality as well as saving supply dollars. ${ }^{14}$

The literature on value analysis is severely lacking and data are hard to come by; however, there are numerous case studies documenting savings through value analysis. Since 1997, the University HealthSystem Consortium (UHC) VAP has documented more than $\$ 500$ million in savings for participating organizations, assisted members in realizing more than \$2 million in cost savings annually, and delivered a return on investment of 10:1 for many members, including University of Mississippi Health Care (UMHC). ${ }^{15}$ The return on investment for a VAP is the ratio of savings (including cost avoidance) the VAP generates for the facility to the total cost of the VAP.

\section{Value analysis program at University of Mississippi Healthcare}

$\mathrm{UMHC}$ is the only level one trauma center in the state of Mississippi and established its VAP in 2008. The VAP exists to assist UMHC in diminishing costs and expanding performance while sustaining or enhancing quality patient care. The mission of the program is to decrease cost through cost-effective standardization and utilization of services, processes, and products, while sustaining and/or improving the quality of patient care.

The goal of the VAP at UMHC, which is a seven-step process, is to improve and/or maintain the quality of patient care at the lowest total delivered cost. It is through value analysis that total expenditure is managed effectively, enabling health care providers to deliver high quality care leading to improved patient outcomes. UMHC has a VAP dedicated to increasing efforts to attain optimal pricing, and standardization and utilization of supplies, services, and processes. The VAP is centered on organizing clinical supply utilization and standardization in order to ensure improved revenue capture for high-cost supplies. UMHC spends approximately $\$ 140$ million dollars on supplies each year according to the most recent estimate. UMHC has the opportunity to standardize and maximize utilization of many patient supplies, services, and processes, as well as to create an environment of product cost awareness and accountability across the campus.

The value analysis process at $\mathrm{UMHC}$ is comprised of seven steps. When a new request is received, the group purchasing organization (GPO) status is reviewed. Mississippi state laws require the UMHC to purchase through its GPO. While there are a few exceptions which include a dollar threshold of less than $\$ 5000$, standardization, sole source items, and compatibility, most instances require purchases to be affiliated with a GPO contract. Therefore, if the requested item is not on the GPO, then value analysis verifies that comparable items are available through the GPO. If comparable items are available via the GPO, then the requestor must examine those items first. The next step is to obtain and review the Food and Drug Administration approval for the requested item. It is important to understand the indication for the item, and if the requestor intends to use the item off-label. Any available literature is reviewed during the second step. Peer-reviewed literature is the gold standard, but in the absence of this, white papers are accepted. The absence of peer-reviewed literature and any ongoing clinical trials are noted as well. Next, value analysis examines patient outcomes. Value analysis considers whether this item decreases the average length of stay, morbidity, mortality, infections, and operative time. Value analysis also ensures that these outcomes are documented in the literature. If outcomes are not documented, then a trial is conducted on 
the device, and outcomes in our facility are noted. Next, value analysis surveys other academic medical centers to obtain their feedback. Value analysis specifically questions if the requested item has been reviewed in their facility, if it was implemented, why or why not, and inquires about any lessons learned during the implementation process. During step five, value analysis confirms an internal justification to determine if the item is truly needed in our facility. Value analysis reviews best practices and standards of care. If the UMHC has not already implemented best practices, then they are implemented first before turning to a specialty product. However, there are times when the requested item is considered best practice, and it is the policy of UMHC to implement best practices. Therefore, if there is a need for the requested item, then the next step is to perform a financial analysis. Value analysis reviews the cost per procedure, contribution margin, and payer mix. Value analysis also considers any savings as a result of a decreased length of hospital stay or operative time, as well as any cost avoidance achieved, such as a reduction in nosocomial infections. The last step is to examine any value added programs the vendor may offer. Value added programs include but are not limited to continuing education, indigent care programs, rebate incentive offerings, clinical trials, and "go green" (environmentally friendly) initiatives.

\section{Materials and methods}

Formed in 1984, UHC (Oak Brook, IL) is an alliance of 107 academic medical centers and 232 of their affiliated hospitals, representing approximately $90 \%$ of the nonprofit academic medical centers in the US. The mission of UHC is to advance knowledge, foster collaboration, and promote change to help members succeed in their respective markets. UHC offers an array of performance improvement products and services.

The clinical data base/resource manager (CDB/RM) provides an expanded set of comparative data and analytic tools to support the clinical operations of member institutions.
Opportunities for improvement are identified by combining patient encounter level and line-item transactional detail to yield information on patient outcomes and high-impact resource utilization. Participating hospitals can benchmark openly with other hospitals by accessing this transparent, web-enabled database. The UHC clinical database fast track product has met the criteria for inclusion in the accreditation process and is included on The Joint Commission's list of acceptable vendors. The UHC clinical database fast track product is committed to meeting future criteria established by The Joint Commission.

This study utilized the UHC CDB/RM as secondary data. The service lines selected for this study, ie, cardiology, cardiovascular, neuroscience, and orthopedics, represent the majority of physician preference items. Academic medical centers included were those who utilize UHC as their core measure vendor. Only academic medical centers having medical schools with accredited residency programs spanning neurosurgery through pediatrics were selected for this study. Excluded were affiliate facilities and academic medical centers which do not utilize UHC as their core measure vendor. Affiliate facilities include county hospitals as well as hospitals without medical schools and accredited residency programs, and only offer resident rotations.

$\mathrm{CDB} / \mathrm{RM}$ data were used to generate reports comparing average length of hospital stay, direct cost, morbidity, and mortality between academic medical centers with and without VAPs. Therefore, two groups were identified, ie, group 1 which had a VAP and group 2 which did not have a VAP.

\section{Statistical analysis}

Continuous variable differences across VAP status were examined using Wilcoxon two-sample tests. Data for the year 2011 were available for descriptive variables at the hospital level, including the Medicare case-mix index, acute care beds, inpatient days, inpatient discharges, inpatient surgeries, intensive care unit cases, and acute transfers (Table 1).

Table I Hospital level characteristics in 201 I

\begin{tabular}{lllll}
\hline & Hospitals $(\mathbf{n}=\mathbf{7 3})$ & No VAP $(\mathbf{n}=\mathbf{3 0}, \mathbf{4 1} \%)$ & VAP $(\mathbf{n}=\mathbf{4 3}, \mathbf{5 9 \%})$ & P value \\
\hline Medicare CMI & $1.93(0.19)$ & $1.89(0.17)$ & $1.97(0.20)$ & 0.088 \\
Acute care beds & $617(226)$ & $608(257)$ & $624.53(206.24)$ & 0.530 \\
Inpatient days & $162,652(61,904.61)$ & $167,382.4(80,030.53)$ & $159,353.2(46,042.45)$ & 0.823 \\
Inpatient discharges & $30,763.18(11,853.96)$ & $32,153.5(15,776.96)$ & $29,793.19(8165.37)$ & 0.982 \\
Inpatient surgeries & $10,266.34(4210.63)$ & $10,602.1(5325.92)$ & $10,032.09(3266.64)$ & 0.866 \\
Percent ICU cases & $19.5(7.47)$ & $17.2(5.29)$ & $21.2(8.36)$ & 0.036 \\
Acute transfers in & $2550.22(1612.23)$ & $2177.1(1497.61)$ & $2810.53(1654.83)$ & 0.116 \\
\hline
\end{tabular}

Note: $* P$ values from Wilcoxon rank-sum tests.

Abbreviations: CMI, case-mix index; ICU, intensive care unit; VAP, value analysis program. 
Primary analyses used multilevel linear mixed model methods to estimate the effects of VAP on primary outcomes (average length of stay, direct cost, morbidity, mortality). Log-transformations were incorporated to account for skewness in the primary outcomes, for which relative difference interpretations are reported by exponentiating linear mixed model parameter estimates. VAP trend effects were estimated using interactions of VAP with study year (Table 2), and are interpreted as relative increases or decreases in the outcome variables per year comparing VAP versus non-VAP hospitals. Stable VAP effects were estimated as a consistent effect across all years in the models without the interaction terms. (ie, the main VAP effect only). Association components of the linear mixed models incorporated random effects at the hospital level, and robust, Huber-White, standard errors were calculated. Sensitivity to inclusion of the adjustment model was conducting by examining unadjusted, fully adjusted (service line, hospital location, all variables included in Table 1), and parsimonious adjusted models (service line, state, Medicare case-mix index, inpatient days, inpatient discharges). "Observed" outcome data models used actual outcome data counts, and "expected" outcome data models used expected counts after incorporating risk adjustment techniques (see Appendix) to account for patientlevel risk factors. VAP models specific for service lines were estimated similarly in the stratified data. Morbidity complications are not modeled, so there would not be an expected complication rate. Study procedures were reviewed and exempted by the University of Mississippi Medical Center institutional review board.

\section{Results}

Seventy-three academic medical centers were included in this study. Forty-one percent $(n=30)$ of the academic medical centers did not have a VAP and 59\% $(n=43)$ did have one. Hospital level characteristics examined include Medicare case-mix index, acute care beds, inpatient days, inpatient discharges, inpatient surgeries, percentage of intensive care unit cases, and acute transfers in. There was no significant difference between the academic medical centers with and without VAPs regarding hospital level characteristics, except for percent intensive care unit cases. Table 1 demonstrates these findings.

Trend differences between VAP and non-VAP were analyzed and no clear pattern favoring either VAP or nonVAP was identified. The trend difference is the difference between VAP and non-VAP slopes per year. If the trend difference estimate is, eg, 0.95 , then at year 1 , VAP would

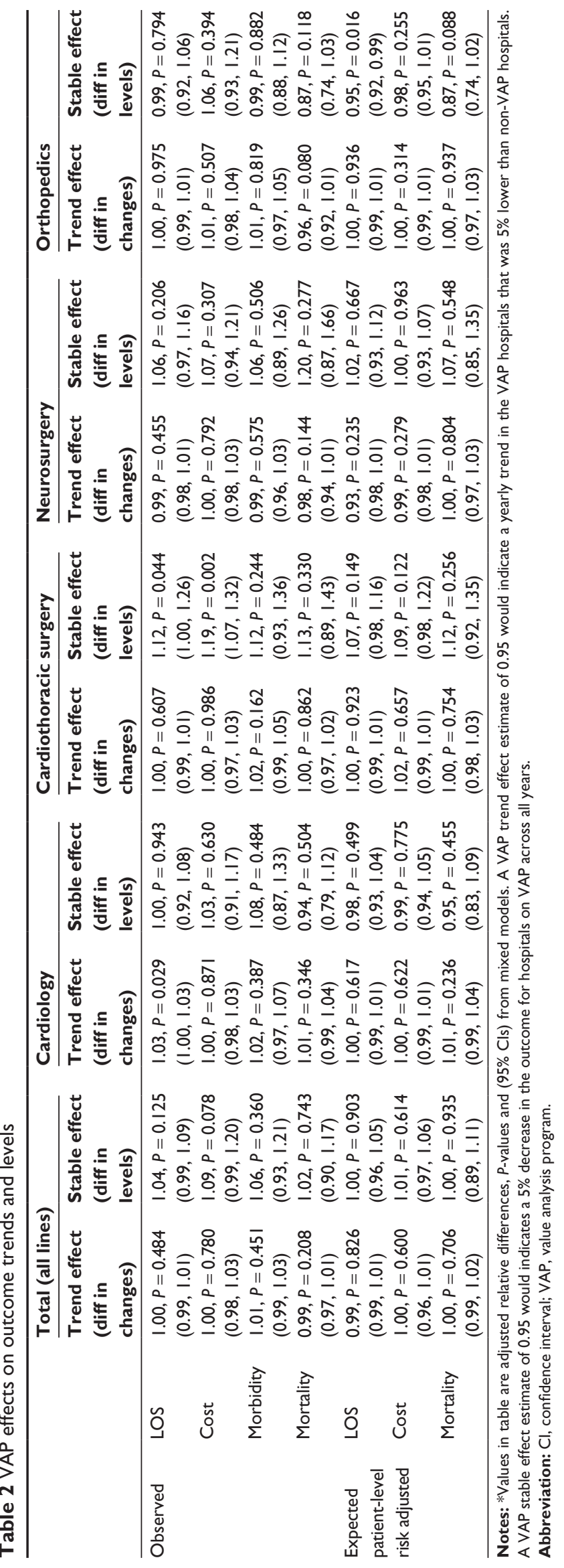


be expected to be 5\% lower, at year 2 we would expect VAP to be $10 \%$ lower, at year 3 it would be $15 \%$ lower, and so on. Alternatively if the trend difference in estimate is, eg, 1.05 , then the VAP is expected to be an additional 5\% higher every year, ie, 5\% higher in year 1, 10\% higher in year 2 , and so on. When differences in the trends were not noted, estimates of the overall stable difference between the groups across all years were calculated. Among the service lines, a significant trend was not found regarding average length of stay, direct cost, morbidity, or mortality for observed or expected outcomes. Similar findings were noted for each of the service lines (Table 2). In summary, significant differences were not noted concerning average length of stay, cost, morbidity, and mortality in academic medical centers with and without VAPs.

\section{Discussion}

One of the most significant forces that will revise the US health care system is the transfer from a volume-based payment system to a value-based purchase system. The relationship between quality of care and the price paid for it will characterize value in health care. Therefore, in order to impart value for patients, it is vital that providers generate collaborative approaches that unite solid clinical outcomes at a reasonable cost, because patients will expect to receive the type of quality care for which they paid. Since 1997, the VAP at UHC has documented more than $\$ 500$ million in savings for participating organizations, assisted members in realizing more than $\$ 2$ million in cost savings annually, and delivered a return on investment of 10:1 for many members, including the UMHC. ${ }^{16}$

There are numerous case studies that support documented savings via value analysis. One academic medical center achieves $\$ 1$ million of cost avoidance over 3 years by requiring that all osteobiologic implants be reviewed by a subcommittee by value analysis. ${ }^{17}$ Another academic medical center achieved $\$ 1.73$ million in savings by implementing a spine formulary through value analysis. ${ }^{18}$ Yet another academic medical center was also successful in delivering a savings of $\$ 500,000$ with spine implants through their VAP. ${ }^{19}$ One academic medical center achieved $\$ 587,000$ in cost avoidance for ventilator-associated pneumonia by changing their basic oral care kits. Even though this initiative would increase their supply costs by an additional $\$ 100,000$ per year; 16 ventilator-associated pneumonia cases were eliminated in 6 months, resulting in a cost avoidance of $\$ 587,000$. The facility documented a decrease in the ventilator-associated pneumonia rate of 3.32 per 1000 ventilator days to 1.96 per
1000 ventilator days during a 6-month period. ${ }^{20}$ At UMHC, the VAP delivered a return on investment of 10:1 in its first year, 11:1 in its second year, and 10:1 in its third year. These savings can be reinvested back into the organization for clinical programs, and capital equipment.

This study is not without limitations. While the theoretical framework of value analysis is essentially the same between the facilities that have VAPs, the structure of many VAPs can differ among institutions, along with how value analysis is defined and implemented. It has been suggested that only $10 \%$ of the nation's hospitals actually practice meticulous value analysis, leaving the remaining $90 \%$ to practice alleged value analysis, that consists of standardization, pricing and/ or contract negotiation, and product trials. It is imperative that the VAP can triage opportunities and establish objective criteria for evidence-based evaluations. The value analysis process can also vary between facilities that maintain VAPs. The effectiveness of a VAP is related to the support of the executive leadership of the organization, which can also fluctuate among facilities that possess VAPs. It is considered best practice for VAP to report to a steering committee. The steering committee serves to ensure accountability throughout the organization, set strategic goals for value analysis teams, and enforce the policies and procedures of the VAP. The maturity of the VAP may also have an effect on outcomes. While it is possible that the academic medical centers identified as not having a VAP may practice some principles of value analysis under a different title according to UHC best practices, they are not likely to be successful without executive support, clinician engagement, and a formal program. The effect of this on the study would be negligible. Savings could not be compared between academic medical centers with and without VAPs. There is no way to obtain a record of any savings in academic medical centers without formal VAPs. Furthermore, direct costs include variables such as labor and fringe benefits, which value analysis does not impact and cannot account for. While direct costs do not demonstrate the impact of value analysis, it was the only cost available to measure. Furthermore, this study was limited to only four service lines, ie, cardiology, cardiothoracic surgery, orthopedics, and neuroscience. Many more service lines exist at academic medical centers.

The impact of value analysis regarding patient outcomes is unclear. The results of this study did not reveal a significant difference in average length of stay, direct cost, morbidity, and mortality between academic medical centers with and without VAPs, but the study findings did not find a negative impact on patient care as a result of VAPs. Stuebing and Miner 
demonstrated a significant savings for one particular hospital via resource utilization. The goal of the 11-week intervention was to reduce the number of laboratory tests performed. As a result, the dollar amount saved was \$54,967 over the 11 -week time period. It is important to note that patient outcomes including morbidity, mortality, readmissions, and average length of stay were not negatively impacted as a result of reducing the number of laboratory tests performed. ${ }^{20}$ Another study at a teaching facility found similar results. By focusing on resource utilization of laboratory testing, a savings of \$2 million was achieved across a 3-year period. Again, morbidity, mortality, average length of stay, and readmissions were not negatively impacted as a result. ${ }^{21}$

In addition to converging on the balance between the business savings side of health care and patient outcomes, value analysis examines which products and/or technologies that are truly beneficial and possibly affect patient outcomes; however, there are multiple factors which impact patient outcomes. Medical errors, comorbidities, acuity level, patient compliance, socioeconomic status, geographic location, and access to health care are just some of the factors that can affect patient outcomes. It is possible that unknown comorbidities went undetected, and therefore had an effect on clinical outcomes. Human involvement also plays a role in patient outcomes. Processes, products, and technologies may provide a framework upon which to assemble a VAP, but human infrastructure is essential to patient outcomes as well. Often successful outcomes may be predicated on who is engaged rather than what is at stake. ${ }^{21}$

On September 22, 2011, UHC in Chicago, IL, recognized 10 of its full academic medical center members with the UHC quality leadership award. The award is given to academic medical centers that demonstrate excellence in delivering high-quality care, as measured by the UHC Quality and Accountability Study. It is important to note that eight of the 10 recipients of this award have a VAP. In conclusion, many factors contribute to clinical outcomes and the role of value analysis is unclear. However, this study did not find a negative impact on patient care as a result of VAPs. Further studies are needed to explore the benefits of value analysis and its effect on patient outcomes.

\section{Disclosure}

Certain algorithms of the risk-adjusted model regarding the UHC clinical database resource manager are proprietary. The data extracted from the database are not proprietary, but have been deidentified. Therefore, raw data can be provided upon request. There was no financial support for this study.
Publication of the study results was not contingent upon sponsor approval or censorship.

\section{References}

1. The Lawrence D. Miles Value Engineering Reference Center. University of Wisconsin-Madison. Available from: http://wendt.library.wisc.edu/ miles/index.html. Accessed May 13, 2011.

2. Marmor T, Oberlander J, White J. The Obama administration's options for health care cost control: hope versus reality. Ann Intern Med. 2009;150:485-489.

3. Emanuel E. Essential elements of a technology and outcomes assessment initiative. JAMA. 2007;298:1323-1325.

4. Ford EW, Scanlon DP. Promise and problems with supply chain management approaches to health care purchasing. Health Care Manage Rev. 2007;32:192-202.

5. Singh A, Schneller ES. The role of the physician executive in managing the health care value chain. Hosp Q. 2000;4:38-43.

6. Murphree J. Value analysis: an Rx in healthcare. Purchasing Today. 2000:55.

7. Yokl R. Strategic value analysis: saving beyond price. Strategic Value Analysis Healthcare. 2007:4271.

8. [No authors listed]. Value in health care: current state and future directions. Healthcare Financial Management Association. Jun, 2011. Available from: http://www.hfma.org/HFMA-Initiatives/Value-Project/ Value-in-Health-Care--Current-State-and-Future-Directions/. Accessed June 24, 2012.

9. Montgomery KS, Schneller E. Hospitals' strategies for orchestrating selection of physician preference items. Milbank Mem Fund $Q$. 2007;85:307-335.

10. Kirschner N. Information on cost-effectiveness: an essential product of a national comparative effectiveness program. Ann Intern Med. 2008; 148:956-961.

11. Coye MJ, Kell J. How hospitals confront new technology. Health Aff (Millwood). 2006;25:163-173.

12. Thorpe KE. The rise in healthcare spending and what to do about it. Health Aff (Millwood). 2005;24:1436-1445.

13. Bodenheimer T. High and rising health care costs. Part 2: technologic innovation. Ann Intern Med. 2005;142:932-937.

14. University HealthSystem Consortium. University of Arkansas for Medical Sciences Achieves \$587,000 Cost Avoidance in VentilatorAssociated Pneumonia. Oak Brook, IL: University HealthSystem Consortium; 2008.

15. University HealthSystem Consortium. The UHC Value Analysis Program: Achieve Cost Efficiencies in Health Care. Oak Brook, IL: University HealthSystem Consortium; 2011.

16. University HealthSystem Consortium. UK HealthCare (UKHC) Achieves Approximately \$1 Million Annual Cost Avoidance through Osteobiologic Implant Management Process. Oak Brook, IL: University HealthSystem Consortium; 2007.

17. University HealthSystem Consortium. UHC Template a Critical Ingredient in \$1.7 Million Spinal Implant Savings for Virginia Commonwealth University Health System. Oak Brook, IL: University HealthSystem Consortium; 2011.

18. University HealthSystem Consortium. Physician Involvement Key to UCLA Healthcare's \$500,000 Annual Spine Savings. Oak Brook, IL: University HealthSystem Consortium; 2007.

19. O’Connor CJ. Supply Chain Strategies and Solutions: Value Analysis, Bringing Science to the Business of Healthcare. Chicago, IL: Association for Healthcare Resource Materials Management; 2011.

20. Stuebing EA, Miner TJ. Surgical vampires and rising health care expenditure: reducing the cost of daily phlebotomy. Arch Surg. 2011; 146:524-527.

21. Attali M, Barel Y, Somin M, et al. A cost-effective method for reducing the volume of laboratory tests in a university-associated teaching hospital. Mt Sinai J Med. 2006;73:787-794. 


\section{Appendix}

\section{Risk adjustment methodology for the clinical database}

The risk adjustment process used in the clinical database involves four steps: assignment of a severity of illness level and a risk of mortality to each case; selection of a patient population to serve as the basis of the model (provide norms); use of multiple regression techniques to predict length of stay, cost, and probability of mortality based on the normative patient population; and assignment of an expected length of stay, cost, and probability of mortality to every patient in the database.

\section{Assignment of severity of illness level}

Patient characteristics, such as severity of illness and age, impact resource utilization, and clinical outcome greatly. To ensure equitable interhospital comparison of resource use and outcomes, one must adjust for differences in patient characteristics. A number of patient classification schemes have been developed to address the issue of patient severity. While each of these schemes defines severity differently, most are similar in one respect, ie, they use specific combinations of primary and secondary diagnoses to define different levels of severity and complexity of treatment.

The all-patient refined diagnosis-related group (APRDRG) grouper, developed by $3 \mathrm{M}$ Health Information Systems, is used by the University HealthSystem Consortium (UHC) to assign a level of illness severity and a risk of mortality level for each patient in the clinical database. The APR-DRGs were developed as an expansion of the DRG system to address patient severity of illness and risk of mortality, as well as resource intensity. The development process for the APR-DRGs involved an iterative process of formulating clinical hypotheses and then testing the hypotheses with historical data. Separate clinical models for severity of illness and risk of mortality were developed for each of the base APR-DRGs and then tested with historical data to review the clinical hypotheses.

In the APR-DRG system, a patient is assigned three distinct descriptors, ie, the base APR-DRG (eg, APR-DRG 044 intracranial hemorrhage or APR-DRG 194 heart failure), severity of illness (SOI) subclass, and risk of mortality (ROM) subclass.
The underlying clinical principle of the APR-DRGs is that the SOI or ROM subclass is largely dependent on the patient's underlying problem and that patients with a high SOI or ROM are usually characterized by multiple serious illnesses. Determination of SOI and ROM is disease-specific, ie, the significance attributed to comorbid conditions is dependent on the underlying problem. High SOI and ROM are primarily determined by the interaction of multiple illnesses involving multiple organ systems which result in patients who are difficult to treat and tend to have poor outcomes. The four SOI subclasses and the four ROM subclasses are minor, moderate, major, and extreme.

Although the subclasses are numbered sequentially, the numeric values represent categories and not scores. It is not meaningful to average the numeric values of the SOI or ROM subclasses across a group of patients to compute an average severity score. The SOI or ROM subclasses can be used as categories within which average values for a measure, such as length of stay (LOS) or cost, are calculated to represent an expected value for that SOI or ROM subclass.

\section{Identification of patient population for model generation}

In order to identify the "mainstream" population to be used for generation of LOS and cost models, several types of cases are excluded:

- incomplete records and "bad" data (defined as cases in DRG 470, ungroupable).

- extreme LOS outliers (defined as cases with LOS greater than the DRG-specific 99th percentile)

- early deaths (defined as death within 2 days of admission)

- early transfers (defined as transfers out within two days of admission)

- cases with potentially avoidable complications.

The UHC complication profiler is used to identify potentially avoidable complications that were not present at the time of admission. The UHC complication profiler is an implementation of research originally conducted at Beth Israel Hospital in Boston, MA, and funded by the Agency for Health Care Policy and Research, through which Iezzoni et al found that using computers quality screening can be performed using ICD-9-CM codes from standard administrative databases. 
The UHC complication profiler first classifies patients into risk pools based on their combination of DRG and ICD9-CM diagnosis and procedure codes (a list of the ICD-9-CM codes and DRGs constituting each risk pool can be found in the UHC complication profiler online documentation). The risk pools, which are broad groupings representing similar levels of invasiveness or technical approaches, identify patients at risk for specific types of complications. The four risk pools are major surgery DRGs, minor surgery DRGs, cardiac procedures, and gastrointestinal endoscopy and endoscopic retrograde cholangiopancreatography.

Although a patient can be in more than one risk pool, each case can be counted only once within a single risk pool. Additionally, patients are at risk only for those complications related to their particular risk pool(s). Prior to release of the current UHC complication profiler, the first criterion for inclusion in the major or minor surgery risk pools was that the date of the primary procedure was less than 2 days after the admission date. This criterion eliminated many cases from these risk pools and, therefore, from assessment of the presence of all complications for those patients. This requirement that the primary procedure not be delayed was meaningful for some complications, such as nosocomial pneumonia following a procedure, or postoperative stroke where the condition could have been developing prior to the procedure rather than post procedure. The requirement was not meaningful for other complications where the length of time prior to the procedure would not be expected to affect the occurrence of the complication during or after surgery, eg, procedure-related perforation or laceration, post-procedure hemorrhage or hematoma. The UHC complication profiler was modified to remove the requirement that the primary procedure date be less than two days after admission from the major and minor surgery risk pools. For specific complications where this requirement is meaningful for determining whether a procedure-related complication occurred, the requirement was added to the algorithm for that complication as an initial decision point.

The UHC complication profiler screens for 25 potentially avoidable complications based on patient procedures and diagnoses (a more thorough description including algorithm diagrams and specific ICD-9-CM codes used by the UHC complication profiler is included in the UHC complication profiler online documentation). The complications screened for and their risk pool assignments are as follows:

\begin{tabular}{ll} 
Complication & Risk pool \\
\hline I. Postoperative stroke & A, B \\
2. Aspiration pneumonia & A, B \\
3. Postoperative pulmonary compromise & A, B \\
4. Postoperative gastrointestinal hemorrhage or & A, B \\
ulceration & \\
5. Postoperative urinary tract complications & A, B \\
6. Cellulitis or decubitus ulcer & A, B \\
7. Septicemia & A, B \\
8. Postoperative or intraoperative shock due to & A, B \\
anesthesia & A, B \\
9. Reopening of surgical site & A, B \\
10. Mechanical complications due to device or implant & A, B \\
II. Miscellaneous complications of procedures & B \\
12. Shock or cardiorespiratory arrest & A, B, D \\
13. Complications relating to central or peripheral & \\
nervous system & A, B, D \\
14. Postoperative acute myocardial infarction & A, B, D \\
15. Postoperative cardiac abnormalities except acute & \\
myocardial infarction & A, B, D \\
16. Postoperative infections except pneumonia and wound & A, B, D \\
17. Procedure related perforations or lacerations & A-D \\
18. Postoperative coma or stupor & A-D \\
19. Nosocomial pneumonia following procedure & A-D \\
20. Postoperative physiologic and metabolic derangements & A-D \\
21. Complications relating to anesthetic agents & A-D \\
22. Venous thrombosis and pulmonary embolism & A-D \\
23. Wound infection & A-D \\
24. Post-procedural hemorrhage or hematoma & \\
25. Other complications of procedures & \\
\hline &
\end{tabular}

For generation of mortality models, only cases flagged as "bad data" and transfers out to another acute care hospital are excluded from the model population.

\section{Regression models}

Regression models are generated to predict LOS, cost, and probability of mortality (dependent variables). Ordinary leastsquares linear regression models are developed for cost and LOS. Because LOS and cost are not normally distributed, a logarithmic transformation is performed to normalize the distribution for these dependent variables prior to model generation. When the model results are used to calculate patient-level expected values, LOS and cost are returned to their nontransformed values and a statistical adjustment to correct for bias in the predictor value is applied. Logistic regression models are constructed for the binary outcome, variable, ie, inhospital mortality. Separate models are built 
for each DRG and base MS-DRG and for each dependent variable.

Strict exclusion rules are applied before building the regression models. For the outcome variable of inhospital mortality, only DRGs and base MS-DRGs with more than 100 cases and an incidence rate greater than $1 \%$ are modeled. Due to the potential for serious estimation problems related to a sparse contingency table, another layer of exclusion criteria is also imposed, ie, the observed incidence counts (rate multiplied by number of cases) must be greater than 50. For LOS and cost, only DRGs and base MS-DRGs with more than 100 "mainstream" cases (ie, cases remaining after exclusion of incomplete records/ bad data, LOS outliers, early deaths, early transfers, and cases with potentially avoidable complications) are modeled. Independent variables included in all the regression models are:

- SOI subclass (from APR-DRG grouper) for LOS and cost; ROM subclass for mortality, ie, patient age, gender, race, admit source (transfer from another acute care hospital, transfer from skilled nursing facility, long-term care facility), low socioeconomic status (based on Medicaid, self-pay, charity as primary payer), and admit status (emergency)

- Comorbid conditions defined by the Agency for Healthcare Research and Quality based on research by Elixhauser et al (ICD-9-CM code definitions for each comorbid condition are available in the online documentation), ie, congestive heart failure, valvular disease, pulmonary circulation disorders, peripheral vascular disease, hypertension (complicated and uncomplicated), paralysis, other neurological disorders, chronic pulmonary disease, diabetes (complicated and uncomplicated), hypothyroidism, renal failure, liver disease, peptic ulcer disease excluding bleeding, acquired immune deficiency syndrome, lymphoma, metastatic cancer, solid tumor without metastasis, rheumatoid arthritis/collagen vascular diseases, coagulopathy, obesity, weight loss, fluid and electrolyte disorders, blood loss anemia, deficiency anemias, alcohol abuse, drug abuse, psychoses, depression.

Additional independent variables are included in the models for specific patient populations. Palliative care identified by ICD-9-CM diagnosis code V66.7 is included in models for conditions such as heart failure, stroke, acquired immune deficiency syndrome, and malignancies. Additional diagnoses and/or procedures specific to certain patient populations, such as neonatology, oncology, transplants, pediatrics, obstetrics, spinal surgery, ventilator support, cardiac surgery, cardiology, neurosciences, and other high impact DRGs and base MS-DRGs are included in the models for those patients. A complete listing of these additional variables and their definitions is available in the online documentation.

Clinical database discharges from major academic medical centers are used to build models. A standard crossvalidation statistical method is used to ensure the stability of the parameter estimates. Data are randomly split into training and validation samples. For LOS and cost models, parameter estimates and prediction errors are examined from the two samples. Only models with stable parameter estimates are used to estimate final predicted values. Key model diagnostics include the c-index, Hosmer-Lemeshow test, and R-square. A C index $\geq 0.70$ is generally accepted as indicating good discrimination by a model. The final regression models are then constructed using the full data set. When a LOS, cost, or mortality model for a DRG or base MS-DRG fails the validation tests, each case in the DRG or base MS-DRG is assigned the average observed value, stratified first by the SOI subclass from the APR-DRG grouper for LOS and cost or ROM subclass for mortality, and then by whether or not the patient was transferred from another acute care hospital.

DRGs and base MS-DRGs with very low observed mortality ( $\leq 1 \%$ or $<50$ deaths) are not modeled for mortality. For those DRGs and base MS-DRGs, expected mortality is based on observed mortality stratified first by ROM subclass within the DRG or base MS-DRG and then by transfer status. For mortality models, goodness of fit tests (eg, Hosmer-Lemeshow tests) are run to determine how well the model predicts the outcome. Only models that "pass" the goodness of fit tests are used to estimate expected probability of mortality.

Potential causes of biased differences between observed and expected values derived from regression models include the following:

- Omitted risk factor(s) - if factors that are significantly related to outcome or resource consumption are not included in the risk adjustment model, the model can underestimate the expected values for hospitals having a disproportionate share of patients with the missing risk factor(s).

- Biased reporting of risk factor(s) - if a hospital consistently underreports or overreports risk factor(s) 
with influential effects on the model estimates, then its expected values will be systematically biased. Given that severity of illness estimation, risk of mortality estimation, complication screens, and comorbidity counts depend on the accuracy of ICD-9-CM coding, review of medical records for accuracy is very important.

- Biased statistical model - if the statistical model is not well calibrated and tested for systematic over or under estimation, then the model may systematically over or under estimate expected values. The literature has shown that this type of bias is not important for analyses at the individual patient level. However, when aggregating to the hospital level, model-related bias may become influential, because institutional differences were not fully accounted for by the model.

To minimize the effects of these factors, hospitals should carefully evaluate coding consistency, completeness, and accuracy, including the coding of UB-92 fields like admission source and admission status, as well as ICD-9-CM diagnosis and procedure coding.

\section{Application of models to entire database}

The output from a stable model includes a coefficient for each of the variables found to be statistically significant predictors of the outcome, ie, LOS, cost, or mortality in the population that was modeled. For LOS and cost, the population modeled is the "mainstream" population without LOS outliers, early deaths and transfers, and potentially avoidable complications. For mortality, the entire clinical databsse population was included in the models. Using the coefficients for each of the statistically significant predictor variables and a flag indicating whether the variable was present in the patient, UHC then calculates a predicted value for the patient. All patients in the database are assigned predicted values for LOS, cost, and mortality regardless of whether they were included in the model generation process or not. The following example illustrates the calculation of expected LOS for a stroke patient (DRG 14).

\section{LOS results for model group}

Number 14 Spec CV dis exc TIA (DRG 14). Model diagnostics: Calculation: $\mathrm{MSE}=0.439$ Validation: $\mathrm{MSE}=0.427, \mathrm{~F}=0.973, P=0.9069$. Final: $\mathrm{R}$-square $=0.200$, Max VIF $=2.842, \operatorname{MSE}=0.430$. Mean observed $=6.208$, mean expected $=6.296$. Cases $=19,377$. Model method $=$ Log-linear regression.

\section{Model results (significant predictors)}

\begin{tabular}{ll} 
Coefficient & Explanatory variable \\
\hline 1.091 & Intercept \\
1.062 & SOI 4 (extreme) \\
0.629 & SOI 3 (major) \\
0.286 & SOI 2 (moderate) \\
0.250 & CC weight loss \\
0.207 & CC fluid and electrolyte disorders \\
0.145 & Admit source (transfer from acute) \\
0.136 & CC psychoses \\
0.117 & CC depression \\
0.104 & CC deficiency anemia \\
0.077 & CC valvular disease \\
0.077 & CC alcohol abuse \\
0.064 & Female, $80-84$ years \\
0.063 & CC hypertension \\
0.056 & Female, $\geq 85$ years \\
-0.036 & CC diabeteswithout CC \\
-0.055 & CC congestive heart failure \\
-0.075 & Race, white \\
-0.245 & CC AlDS
\end{tabular}

Abbreviations: AIDS, acquired immune deficiency syndrome; SOI, severity of illness; CC, chronic conditions.

\section{Characteristics of patient $X$}

Patient $\mathrm{X}$ was an 83-year-old white female transferred from a local community hospital with hypertension, valvular heart disease, and weight loss. The SOI subclass assigned by the APR-DRG grouper was 3 (major). For each of the significant explanatory variables from the model, determine whether that variable was present for this patient. If a variable is present, it receives a value of 1 , if not present its value is 0 :

\begin{tabular}{|c|c|}
\hline Variable & Value \\
\hline SOI 4 (extreme) & 0 \\
\hline SOI 3 (major) & 1 \\
\hline SOI 2 (moderate) & 0 \\
\hline CC weight loss & 1 \\
\hline CC fluid and electrolyte disorders & 0 \\
\hline Admit source (transfer from acute) & 1 \\
\hline CC psychoses & 0 \\
\hline CC depression & 0 \\
\hline CC deficiency anemia & 0 \\
\hline CC valvular disease & 1 \\
\hline $\mathrm{CC}$ alcohol abuse & 0 \\
\hline Female, $80-84$ years & 1 \\
\hline CC hypertension & 1 \\
\hline Female, aged $\geq 85$ years & 0 \\
\hline CC diabetes without CC & 0 \\
\hline $\mathrm{CC}$ congestive heart failure & 0 \\
\hline Race, white & 1 \\
\hline CC AIDS & 0 \\
\hline CC deficiency anemia & 0.104 \\
\hline
\end{tabular}

Abbreviations: AIDS, acquired immune deficiency syndrome; SOI, severity of illness; CC, chronic conditions. 


\section{Calculating expected LOS}

For each of the significant predictor variables, multiply the variable's coefficient from the model times the patient value for that variable:

\begin{tabular}{|c|c|c|c|c|}
\hline Variable & Value & $\mathbf{x}$ & Coefficient & Result \\
\hline SOI 4 (extreme) & 0 & & 1.062 & 0 \\
\hline SOI 3 (major) & I & & 0.629 & 0.629 \\
\hline SOI 2 (moderate) & 0 & & 0.286 & 0 \\
\hline CC weight loss & I & & 0.250 & 0.250 \\
\hline $\begin{array}{l}\text { CC fluid and electrolyte } \\
\text { disorders }\end{array}$ & 0 & & 0.207 & 0 \\
\hline $\begin{array}{l}\text { Admit source } \\
\text { (transfer from acute) }\end{array}$ & I & & 0.145 & 0.145 \\
\hline CC psychoses & 0 & & 0.136 & 0 \\
\hline CC depression & 0 & & 0.117 & 0 \\
\hline CC deficiency anemia & 0 & & 0.104 & 0 \\
\hline $\mathrm{CC}$ valvular disease & I & & 0.077 & 0.077 \\
\hline CC alcohol abuse & 0 & & 0.077 & 0 \\
\hline Female, 80-84 years & I & & 0.064 & 0.064 \\
\hline CC hypertension & I & & 0.063 & 0.063 \\
\hline Female, aged $\geq 85$ years & 0 & & 0.056 & 0 \\
\hline $\mathrm{CC}$ diabetes without $\mathrm{CC}$ & 0 & & -0.036 & 0 \\
\hline $\mathrm{CC}$ congestive heart failure & 0 & & -0.055 & 0 \\
\hline Race, white & I & & -0.075 & -0.075 \\
\hline CC AIDS & 0 & & -0.245 & 0 \\
\hline
\end{tabular}

Abbreviations: AIDS, acquired immune deficiency syndrome; SOI, severity of illness; CC, chronic conditions.

Calculate the sum of the intercept (baseline) value plus all of the individual variable "coefficient $\mathrm{X}$ patient value" results:

Log expected value $=$ Intercept $+(\operatorname{var} 1$ coefficient $X$ var1 $\mathrm{pt}$ value $)+($ var2 coefficient $X$ var2 pt value $)$ $\ldots+(\operatorname{var} n$ coefficient $X$ varn $p t$ value $)$

Log expected value for Patient $\mathrm{X}=1.091+0+0.629+0$ $+0.250+0+0.145+0+0+0+0.077+0+0.064+$ $0.063+0+0+0+-0.075+0=2.244$

The predicted values will be biased by the factor exp $(\sigma 2 / 2)$. To correct for bias when back- transforming log transformed data fit with a log-linear regression the antilog of the predicted value is multiplied by the bias correction factor which is the antilog of 0.5 times the mean square error from the log-linear regression. When the log expected value is converted to its antilog and the correction factor is applied to correct for bias in the predictor value, the expected LOS for this patient is 11.69 days. The next example illustrates the calculation of expected probability of mortality for a stroke patient (DRG 14).

\section{Model group: 14 - Spec CV dis exc TIA (DRG I4)}

Model diagnostics: Calculation, Chi-sq $=20.411$ Validation: Chi-sq $=38.383, \mathrm{~F}=1.881, P=0.1803$, Final: $\mathrm{Max} \mathrm{VIF}=2.789$, Hosmer-Lemeshow $=48.622, P=P<0.001, \mathrm{df}=10$, $\mathrm{C}=0.822$, mean observed $=0.1334$, mean expected $=0.1334$, cases $=21,394$ model method $=$ logistic regression

\section{Model results (significant predictors)}

\begin{tabular}{ll} 
Coefficient & Explanatory variable \\
\hline 3.292 & ROM 4 (extreme) \\
2.856 & Palliative flag \\
1.830 & ROM 3 (major) \\
0.500 & Female, $\geq 85$ years \\
0.489 & Male, $\geq 85$ years \\
0.473 & Female, $80-84$ years \\
0.448 & CC renal failure \\
0.417 & Male, $80-84$ years \\
0.414 & Admit source, emergency \\
0.402 & Admit source, transfer from acute \\
-0.190 & CC diabetes without CC \\
-0.320 & CC hypothyroidism \\
-0.392 & CC valvular disease \\
-0.423 & CC fluid and electrolyte disorders \\
-0.490 & CC CHF \\
-0.609 & CC PVD \\
-0.649 & CC psychoses \\
-0.663 & CC deficiency anemia \\
-0.772 & CC diabetes with CC \\
-0.807 & CC PCD \\
-0.845 & CC depression \\
-1.177 & CC pept ulcer dis $\times$ bleed \\
-1.304 & CC AIDS \\
-1.600 & Male, I-I7 years \\
-1.652 & CC weight loss \\
\hline
\end{tabular}

\section{Characteristics of patient $Y$}

Patient $Y$ was a black male, aged 54 years, transferred from another hospital, emergency admit status, APR-DRG $\mathrm{ROM}=$ extreme, principal diagnosis 430 (subarachnoid hemorrhage), Medicaid, with CC COPD, congestive heart failure, drug abuse, coronary artery disease, asymptomatic human immunodeficiency status, and history of deep vein thrombosis/embolism with long-term anticoagulant use.

For each of the significant explanatory variables from the model, determine whether that variable was present for this patient. If a variable is present it receives a value of 1 , if not present its value is 0 . For each of the significant predictor variables, multiply the variable's coefficient from the model times the patient value for that variable: 


\begin{tabular}{|c|c|c|c|c|}
\hline Variable & Value & $\mathbf{x}$ & Coeff & Result \\
\hline ROM 4 (extreme) & 1 & & 3.292 & 3.292 \\
\hline Palliative care & 0 & & 2.856 & 0 \\
\hline ROM 3 (major) & 0 & & 1.830 & 0 \\
\hline Female, $\geq 85$ years & 0 & & 0.500 & 0 \\
\hline Male, $\geq 85$ years & 0 & & 0.489 & 0 \\
\hline Female, $80-84$ years & 0 & & 0.473 & 0 \\
\hline $\mathrm{CC}$ renal failure & 0 & & 0.448 & 0 \\
\hline Male, $80-84$ years & 0 & & 0.417 & 0 \\
\hline Admit stat emergency & 1 & & 0.414 & 0.414 \\
\hline Admit src trns frm acute & 1 & & 0.402 & 0.402 \\
\hline CC diabetes without $\mathrm{CC}$ & 0 & & -0.190 & 0 \\
\hline CC hypothyroidism & 0 & & -0.320 & 0 \\
\hline $\mathrm{CC}$ valvular disease & 0 & & -0.392 & 0 \\
\hline $\mathrm{CC}$ fluid and electrolytes dis & 0 & & -0.423 & 0 \\
\hline $\mathrm{CC} \mathrm{CHF}$ & 1 & & -0.490 & -0.490 \\
\hline CC PVD & 0 & & -0.609 & 0 \\
\hline CC psychoses & 0 & & -0.649 & 0 \\
\hline CC defic anemia & 0 & & -0.663 & 0 \\
\hline CC diabetes with $\mathrm{CC}$ & 0 & & -0.772 & 0 \\
\hline CC PCD & 0 & & -0.807 & 0 \\
\hline CC depression & 0 & & -0.845 & 0 \\
\hline CC Pept ulc dis exc bld & 0 & & -1.177 & 0 \\
\hline CC AIDS & 0 & & -1.304 & 0 \\
\hline Male, I-I7 years & 0 & & -1.600 & 0 \\
\hline CC weight loss & 0 & & -1.652 & 0 \\
\hline
\end{tabular}

Abbreviations: AIDS, acquired immune deficiency syndrome; $\mathrm{CHF}$, congestive heart failure; coeff, coefficient; PCD, pulmonary circulatory disease; PVD, peripheral vascular disease; $\mathrm{CC}$, chronic conditions.

\section{Formula for calculating expected mortality for a case \\ $N$ expected mortality $=\exp ($ intercept

$$
+\Sigma\left(\operatorname{coeff} i^{*} \operatorname{var} i\right) i=1
$$$$
N 1+\exp \left(\text { intercept }+\Sigma\left(\text { coefficient }^{*} \operatorname{var} i\right) I=1\right.
$$

where $n=\#$ of significant variables or risk factors; var $i=1$ or 0 for variable $i$ present in this patient or not; coeff $i=$ coefficient for variable $i$; exp is the exponential function or $\exp (\mathrm{x})=\mathrm{e}^{\mathrm{x}}[\mathrm{eg}, \exp (0)=1, \exp (1)=2.718]$. Using this formula, patient Y's expected probability of mortality is 0.4960

\section{Model calibration}

To ensure that the models are well calibrated for each of the fiscal years to which they are applied, intercept values and coefficients for each of the variables found to be statistically significant during model generation are recalculated for each fiscal year. There are six sets of model results files (DRG-based LOS, cost, mortality and MS-DRG based LOS, cost, mortality) available online for each fiscal year.

The intercept values and coefficients from the 2007 fiscal year model results files will be applied to 2008 fiscal year data until that fiscal year is completed. At that time, the models will be recalibrated for 2008 fiscal year. This process will then be repeated for each fiscal year in the future.

\section{References}

1. Iezzoni LI, Foley SM, Heeren T, et al. A method for screening the quality of hospital care using administrative data: preliminary validation results. QRB Qual Rev Bull. 1992;18:361-371.

2. Iezzoni LI, Daley J, Heeren T, et al. Identifying complications of care using administrative data. Med Care. 1994;3:700-715.

3. Iezzoni LI, Daley J, Heeren T, et al. Using administrative data to screen hospitals for high complication rates. Inquiry. 1994;31:40-55.

4. Iezzoni LI, Heeren T, Foley SM, Daley J, Hughes J, Coffman GA. Chronic conditions and risk of in-hospital death. Health Serv Res. 1994;29:435-460.

5. Iezzoni LI, Shwartz M, Ash AS, Hughes JS, Daley J, Mackiernan YD. Severity measurement methods and judging hospital death rates for pneumonia. Med Care. 1996;34:11-28.

6. Iezzoni LI, Shwartz M, Ash AS, Mackiernan YD. Does severity explain differences in hospital length of stay for pneumonia patients? J Health Serv Res Policy. 1996;1:65-76.

7. Iezzona LI. Assessing quality using administrative data. Ann Intern Med. 1997;127(8 Pt 2):666-674.

9. Iezzoni LI, editor. RiskAdjustment for Measuring Healthcare Outcomes. 2nd ed. Chicago, IL: Health Administration Press; 1997.

10. Weingart SN, Iezzoni LI, Davis RB, et al. Use of administrative data to find substandard care: validation of the complications screening program. Med Care. 2000;38:796-806.

11. Hosmer DW, Lemeshow S. Applied Logistic Regression. 2nd ed. New York, NY: John Wiley and Sons Inc; 2000.

12.

13. Avrill RF, Goldfield N, Hughes JS, et al. All Patient Refined Diagnosis Related Groups (APR-DRGs) Version 20.0: Methodology Overview. Wallingford, CT: 3M Health Information Systems; 2003.

14. Elixhauser A, Steiner C, Harris DR, Coffey RM. Comorbidity measures for use with administrative data. Med Care. 1998;36:3-5.

\section{Publish your work in this journal}

The Journal of Healthcare Leadership is an international, peer-reviewed, open access journal focusing on leadership for the health profession. The journal is committed to the rapid publication of research focusing on but not limited to: Healthcare policy and law; Theoretical and practical aspects healthcare delivery; Interactions between healthcare and society and evidence-based practices;

\section{Dovepress}

Interdisciplinary decision-making; Philosophical and ethical issues; Hazard management; Research and opinion for health leadership; Leadership assessment. The manuscript management system is completely online and includes a very quick and fair peer-review system. Visit http://www.dovepress.com/ testimonials.php to read real quotes from published authors. 